\title{
Dietary analysis and nutritional behaviour in people with and without age-related macular
}

disease

\section{Abstract}

Background and Aims: Consumption of antioxidant nutrients can reduce the risk of progression of age-related macular degeneration (AMD) - the leading cause of visual impairment in adults over the age of 50 years in the UK. Lutein and zeaxanthin (L\&Z) are of particular interest because they are selectively absorbed by the central retina. The objectives of this study were to analyse the dietary intake of a group of AMD patients, assess their ability to prepare and cook healthy food, and to make comparisons with people not affected by AMD.

Methods: 158 participants with AMD were recruited via the UK charity The Macular Society, and fifty participants without AMD were recruited from optometric practice. A telephone interview was conducted by trained workers where participants completed a 24 hour food diary, and answered questions about cooking and shopping capabilities.

Results: In the AMD group, the average L\&Z intake was low in for both males and females. Those able to cook a hot meal consumed significantly more L\&Z than those who were not able. Most participants were not consuming the recommended dietary allowance of fibre, calcium, vitamin D and E, and calorific intake was also lower than recommendations for their age-group. The nonAMD group consumed more kilocalories and more nutrients than the AMD group, but the L\&Z intake was similar to those with AMD. The main factor that influenced participant's food choices was personal preference.

Conclusion: For an 'informed' population, many AMD participants were under-consuming nutrients considered to be useful for their condition. Participants without AMD were more likely to reach recommended daily allowance values for energy and a range of nutrients. It is therefore essential to design more effective dietary education and dissemination methods for people with, and at risk of, AMD. 


\section{Introduction}

Age-related macular degeneration (AMD) can result in loss of central vision, and is the leading cause of visual impairment in adults (> 50 years) in the UK ${ }^{(1)}$. In 2012, the prevalence of AMD was predicted to increase significantly by 2020 due to aging of the population ${ }^{(2,3)}$.

The Age-Related Eye Disease Study (AREDS) (4) reported that taking a supplement containing vitamins $\mathrm{E}$ and $\mathrm{C}$, beta-carotene and zinc reduced risk of progression of the disease by $25 \%$. Since then, the carotenoids lutein $(\mathrm{L})$, zeaxanthin $(\mathrm{Z}$ ) have been identified as nutrients that can provide a protective role in the progression of AMD due to their antioxidant and photo protective properties ${ }^{(5)}$. Collectively, $\mathrm{L}$ and $\mathrm{Z}$ form the macular pigment which interacts with free radicals and reactive oxygen species, prevent lipid peroxidation and filter out high energy blue light ${ }^{(6)}$. Carotenoids are not produced by the body and must be obtained via the diet. Recently, the AREDS II (7) found that people who took a supplement containing $\mathrm{L}$ and $\mathrm{Z}$ instead of beta-carotene had their risk of progression reduced by a further $18 \%$ compared with the original AREDS formulation ${ }^{(7)}$.

Despite results from AREDS studies, there remains confusion among patients and practitioners in what supplements to take, and what foods should be consumed in order to maximise absorption of useful nutrients ${ }^{(8)}$. Many patients turn to other organisations for clarity of information such as the Macular Society - the UK charity that is devoted to helping those with diseases of the macula. Following the results of AREDS II, the Macular Society have advocated the use of the AREDS II formulation, where appropriate, and eating vegetables that are L\&Z rich. The highest mole percentage of L\&Z has been found to be in egg yolk, maize (corn), spinach, collard greens and kale (9)

Patients who have sought the help of the Macular Society could be considered an 'informed' population as they have information available to them in the form of monthly magazines, written material, a helpline and the Society’s website. However, in a recent study, not all were not taking a nutritional supplement and many of those that did take a supplement were not taking a clinically proven formulation or dosage ${ }^{(10)}$. We therefore sought to investigate this population's dietary intake of $\mathrm{L}$ and $\mathrm{Z}$, and compare it to a cohort of age matched patients without the condition. As dietary patterns are multi-factorial, any other contributing factors will be investigated. 
The objectives of this study were to analyse the nutrient intake of a group of AMD patients and a group of non-AMD patients, and to determine their ability to prepare and cook healthy food.

\section{Materials and Methods}

Using data from a previous study from Bartlett et al, it was calculated that for an average effect size (Cohen's d) of 0.4, a minimum sample size of between 15 and 94 would be appropriate for each cohort. $^{(11)}$

\section{AMD Participants}

A total of 158 participants with AMD were recruited between January 2012 and March 2012. Recruitment was via the Macular Society helpline. Individuals who contacted the Macular Society helpline between January 2012 and March 2012 were asked if they would like to take part in a telephone survey. Inclusion criteria for potential participants were that they should be aged over 55 years and have been diagnosed with any form of AMD.

\section{Non-AMD Participants}

A group of 50 participants without AMD were recruited between August 2013 and December 2013. Recruitment was via seven optometric practices around the UK and Aston University patient clinics. The study was advertised on posters, and individuals who took part volunteered of their own accord and provided contact details and a convenient time to be telephoned. The only inclusion criterion was that they should be aged over 55 years - individuals with co-morbidities and other visual problems were not excluded.

\section{Survey Design}

A 36 question cross-sectional survey was designed to explore nutritional habits, supplement usage, physical abilities in food preparation and cooking, and sources of knowledge in order to ascertain the beliefs participants have, and compare their beliefs with their behaviours. The initial questions covered demographic topics, occupations and participants’ perceptions of the link between nutrition and AMD. The terms 'wet' and 'dry were employed to coincide with many patient's understanding of AMD classifications. After a section on nutritional supplement use, the questions subsequently focused on perceived state of vision and health, and ability to perform preparation and cooking of food. Participants also provided a 24 hour food recall. This was done as part of the telephone survey so the patient had little time to prepare and would be more likely to report honestly. Participants were asked to quantify the amounts of food eaten by using the Zimbabwe Hand method ${ }^{(12)}{ }_{-}$ 
participants used their palms or fingers to estimate the portion size of various foods. The survey was then piloted, refined and administered to the cohort. Full details of the piloting process and survey design and are reported elsewhere ${ }^{(10)}$. The focus of this report is on the dietary aspects of this survey and cooking abilities, hence, not all the results of the 36 questions are covered here ${ }^{(10)}$. The participants' occupations were divided into 10 major groups using the International Standard Classification of Occupations (ISCO) version 08.

The 24 hour food diary data was analysed using nutritional software A La Calc (Red Hot Rails LLP, Doncaster, UK.), where each participant's daily food was analysed for numerous nutrients, calorie values and other constituents using the USDA (United States Department of Agriculture) SR25 food database (

\section{Procedure}

If an AMD patient decided to participate, oral informed consent was obtained over the telephone and they were advised that they could withdraw at any time. An appointment was scheduled for a future telephone interview or the interview began immediately if the AMD patient agreed. NonAMD patients who provided their contact details were telephoned at a time that they specified was convenient, and the interview usually began immediately. The survey typically lasted 25 minutes and was administered either by one of four Macular Society employees who were trained by RS or by RS. All responses were recorded using Bristol Online Survey software (University of Bristol, Bristol, UK) ${ }^{(10)}$.

\section{Data and Statistical Analysis}

Descriptive analyses were performed using the software Microsoft Excel. Data was then analysed in statistical software IBM SPSS version 20 (IBM UK Ltd, Portsmouth, Hampshire) to draw comparisons between results using parametric and non-parametric tests as not all the data was normally distributed.

\section{Ethics}

This study was conducted according to the guidelines laid in the Declaration of Helsinki and all procedures involving human subjects were approved by the Aston University Ethics Committee. Verbal informed consent was obtained from all subjects and formally recorded. 


\section{$\underline{\text { Results }}$}

125

126

127

128

129

130

131

132

133

134

135

136

137

138

139

140

141

142

143

144

145

146

147

148

149

150

151

152

153

154

\section{Sample Characteristics: AMD Participants}

Table 1 shows some of the demographic characteristics of the sample; AMD participants were aged 56-95 (mean $79 \pm$ sd 7.8 years). Of the AMD cohort, 61\% were female, with both sexes showing similar age distributions. The prevalence of 'wet' and 'dry' types of AMD was almost equal. The mean duration of the disease was $6.08 \pm 4.7$ years (median 5 years, range 1 to 25 years). The majority of AMD participants (63\%) were not registered sight impaired (partially sighted) or severely sight impaired (blind). There was a trend for participants who were on a visual impairment register to have had AMD for a longer time period (Mann-Whitney $U=977.5, p=0.07$ ). No AMD participant felt that their vision was "extremely good" on the day of the interview - $57 \%$ of participants felt their vision was "poor" or "extremely poor" and only 7\% felt their vision was "good". These results contrast with perceptions of general health in that only 21\% of AMD participants felt their general health was poor, and $41 \%$ felt their health was good. For these reasons, the sample was considered to be a healthy population whose only health issue was their visual status.

\section{Sample Characteristics: Non-AMD Participants}

Non-AMD participants were aged 55-89 (mean $67 \pm 8.0$ years). Of the non-AMD group, 70\% were female, with both sexes also showing similar age distributions. On the day of the interview, the majority of the non-AMD participants felt their vision was either "extremely good" (14\%), or "good" (46\%). No participant felt their vision was "extremely poor". 70\% of the non-AMD participants reported they felt their general health was either "good" or "extremely good". This cohort can also be considered a healthy population

The cohorts have some similarities in the reported characteristics. A high percentage of participants in both cohorts lived in their own home, and only a few lived in sheltered accommodation. However, half of the AMD participants lived with their partner, with just under half living alone, but in comparison, fewer of the participants without AMD lived alone and more lived with their partner. Also, more participants in the AMD cohort answered that they felt their general health was 'poor' compared to the participants without AMD. Unsurprisingly, the largest difference between the cohorts was the self-reported visual ability, with many of the AMD cohort reporting 'poor' vision. 
Because the non-AMD participants were generally younger than the AMD group (mean 67 years, versus 79 years), a sub-group of age-matched AMD participants (49 participants in total) were used to compare data with the non-AMD cohort.

\section{Table 1 inserted about here.}

\section{Dietary Analysis}

Table 2 displays the results of the 24 hour food diary data for the AMD and non-AMD participants. The mean amounts of certain nutrients or energy consumed for females and males in the cohort are displayed, together with the recommended dietary allowances (RDA) for each constituent, as recommended mainly by the UK FSA (Food Standards Agency http://multimedia.food.gov.uk/multimedia/pdfs/nutguideuk.pdf) for those aged over 50 years. Table 2 shows that in the AMD cohort, both men and women fail to meet the daily RDA for many nutrients such as fibre, calcium, vitamin E, folic acid and carbohydrates. Of particular interest is the finding that in the AMD cohort, both genders are failing to reach the $10 \mathrm{mg}$ amount of daily L\&Z recommended by the Macular Society. Although not statistically significant, there was a trend that those who were on a sight impairment register consumed more L\&Z than those AMD participants who were not on any type of register (Kruskal Wallis $\mathrm{H}=4.951, \mathrm{p}=0.08$ ), and females appeared to consume more L\&Z than males in the AMD group. One male participant diet data was excluded as he had unusually consumed a large amount of kale, and the results were slightly skewed (the average with this participant included is listed in the table in parenthesis). L\&Z consumption was compared with the type of AMD the participants had (dry vs. wet), occupation, and the number of years they had the condition, but no trends were apparent. The amount of L\&Z was also low in both genders of the non-AMD group, but a little more equally.

The largest difference between the two cohorts was the number of calories consumed. The number of calories consumed by the AMD cohort was significantly lower than the daily RDA for each gender in this age group. In contrast, females in the non-AMD cohort surpassed the calorific RDA, and males in the non-AMD cohort also consumed nearer to the RDA. This difference in calorie intake between the AMD and non-AMD cohorts is statistically significant (independent t-test $\mathrm{F}=$ 19.2, $\mathrm{p}=0.00$ ). Other nutrient differences between the cohorts include carbohydrates, protein, fat, fibre and calcium - participants with AMD consumed less compared to participants without AMD.

There were no significant gender differences found with respect to the amount of L\&Z consumed, the number of calories consumed, the ability to cook a hot meal or supplement use in either cohort 
(Kruskal-Wallis and Mann-Whitney U non-parametric tests). Calorie intake was also compared with the age of participants and living arrangements in each cohort, but no significant results were found. The characteristics between the two groups were also analysed, but the differences between them were not found to be statistically significant (Independent t-test).

\section{Table 2 inserted about here.}

Many participants in both cohorts ate only a small variety of vegetables: 140 participants listed carrots, 116 listed peas, 115 listed broccoli. Other popular choices were cabbage (90 participants) and spinach (49 participants). Interestingly only 25 AMD participants ate kale which is considered to be one of the most lutein rich vegetables. No participant in the non-AMD cohort ate kale at all. Interestingly, $85 \%$ of AMD participants ate mostly raw vegetables on the day of the interview, but $62 \%$ of non-AMD participants ate cooked vegetables on the day of the interview. $10 \%$ of the AMD participants and $8 \%$ of the non-AMD participants did not eat any vegetables at all on the interview day.

\section{Food preparation and cooking}

The majority of participants reported they could prepare (65\% AMD, 74\% non-AMD) and cook (68\% AMD, 72\% non-AMD) their own food, with participants citing that their partner prepared (24\% in both cohorts)/ cooked (26\% in both cohorts) food for them. When asked if participants were able to cook a hot meal by themselves if they had to, $81 \%$ of AMD and $96 \%$ of non-AMD participants reported that they could. AMD participants who were not able to cook a hot meal $(n=29)$ consumed significantly less $L \& Z(0.85 \pm 0.72 \mathrm{mg})$ than those who were able to cook a hot meal $(1.68 \pm 2.35 \mathrm{mg})$ (Mann-Whitney $\mathrm{U}=1240.5, \mathrm{p}=0.007)$. Figure 1 shows that many AMD participants feel that they are able to cook and prepare food and that there are no barriers to changing their diets other than that they do not wish to.

\section{Figure 1 inserted about here.}

Fifty six percent of AMD and 86\% non-AMD participants are self-sufficient in carrying out their own food shopping; whilst for $28 \%$ of AMD respondents and 14\% of non-AMD respondents, a family member did this task for them. Less AMD participants are therefore self-sufficient than nonAMD participants, and this will impact on food choices and food consumption. The remainder of the respondents utilised other methods to obtain their food needs such as Meals-On-Wheels (Social 
Services) or friends. Participants were asked to identify all of the sources from which they acquire food. Supermarkets were reported by 135 participants, and 36 also reported they purchased food from a local shop. Some 12 participants harvest their own food. Only four AMD participants relied upon delivered ready meals such as Meals-On-Wheels. The main reason for selecting the type of food appears to be preference (44\% AMD, 46\% non-AMD), secondary to the effect on health (34\% both cohorts). Only 4\% of the AMD group, and $8 \%$ of the non-AMD group listed 'habit' as an influencing factor.

\section{Age-matched comparisons between AMD and non-AMD participants}

Because the AMD participants were older than the non-AMD participants, it was appropriate to compare the non-AMD group with an age-matched sub-group of AMD participants $(n=49)$. The mean age of the sub-group was 68.8 years, and this did not differ significantly from the AMD group (Spearman 0.063, p=0.662). Eighty-six percent of the group were female. There were a higher percentage of participants in the sub-group that felt that their health was 'extremely good' (16\%) and this is close to the percentage of non-AMD participants ( $22 \%$ ).

Table 3 shows the mean diet results of the aged-matched sub-group of AMD participants, with the overall mean diet results from the non-AMD cohort alongside. In this comparison, the difference between the AMD participants and the non-AMD participants is still substantial; the non-AMD group consumed more of each nutrient analysed than the AMD age-matched sub-group. The average energy intake for AMD participants was 1502 kilocalories compared to 2198 kilocalories consumed by non-AMD participants (paired t-test $\mathrm{p}<0.001$ ). However, the exception to this rule was the L\&Z intake, where the average consumption was not significantly different (1.8 mg in the AMD group versus $1.5 \mathrm{mg}$ in the non-AMD group).

Table 3 inserted about here.

\section{$\underline{\text { Discussion }}$}

This sample of people with AMD consumed an average of $1.4 \mathrm{mg}$ of $\mathrm{L} \& \mathrm{Z}$. This is below the $10 \mathrm{mg}$ daily amount considered to be required for augmentation of macular pigment ${ }^{(13)}$. In addition, they were not attaining the RDA of other nutrients such as vitamin E and calcium. Those that were not able to cook a hot meal by themselves consumed significantly less L\&Z than those that were able to cook a hot meal. Participants were not consuming enough calories for their age group, and were adhering to a diet with little variety. Many participants were not consuming the L\&Z-rich 
vegetables, such as spinach and kale. A sample of non-AMD patients consumed calories and other nutrients much nearer to the RDA, and consumed an average of $1.5 \mathrm{mg}$ of L\&Z.

In terms of differences and similarities between the AMD participants and the non-AMD participants, the demographic characteristics were similar. Both groups ate a limited variety of foods, and consumed similar amounts of L\&Z, indicating that AMD participants were not consuming much more than those without the condition. However, the amount of nutrients and energy consumed was quite different between the groups, as the non-AMD cohort consumed much more than the AMD group and in some cases even more than the RDA.

The majority of AMD participants felt their vision was poor on the day of the interview. However, this visual impairment did not impact on their kitchen abilities as most participants felt able to cook a hot meal, go shopping and obtain food from a supermarket themselves. Many felt that their vision or physical capability was not preventing from changing their diet should they wish to; many did not want to change their diet. The main factor that influenced both cohort participants' food choices was preference, indicating that participants would only eat food if they liked it.

Since the AMD cohort all had a form of AMD, and were motivated to contact the Macular Society for assistance, it may be presumed that they were interested in preventing the disease from progressing. All will have received diet advice from the Macular Society, if not from their ophthalmologist. Overall, however, participants were not consuming enough of the majority of the nutrients recommended, particularly L\&Z. The reasons behind this are likely to be multi-factorial dislike for the nutrient-rich foods, lack of knowledge of how to cook or prepare them, misjudging the amount required per day, or lack of control (family or caregivers cook food). We propose that the most likely reason is because dietary habits are difficult to change - especially with increased age ${ }^{(14)}$. L\&Z intake was associated with those who were able to cook a hot meal, indicating that those who were more proficient in the kitchen were able to consume these foods more easily.

There does not seem to be evidence that the information that participants had received from the Macular Society or other sources modified their behaviour. Ley's model on effective communication in medical practice ${ }^{(15)}$ stresses the importance of memory next to factors such as the understanding of information and satisfaction with the treatment. Indeed, $40-80 \%$ of medical information provided by healthcare practitioners is forgotten immediately ${ }^{(16)}$. The greater the amount of information presented, the lower the proportion correctly recalled ${ }^{(17)}$ furthermore, almost half of the information that is remembered is incorrect . Studies have proposed three explanations for memory loss-first, practitioner factors, such as use of medical jargon; second, the type of 
information (e.g. verbal, written); and, third, patient factors, such as low education or specific expectations $^{(16)}$.

With respect to medical information, an inverse relationship has been reported between age and amount of information correctly recalled ${ }^{(16)}$. However, although older adults have difficulty spontaneously recalling medical information, they are able to take advantage of cues to access verbally learned information ${ }^{(18)}$. Studies into patient compliance have shown that patients rarely adhere fully to practitioner advice ${ }^{(19)}$, and AMD patients are reported to have not complied with recommended dietary supplement advice ${ }^{(10,20)}$.

Many patients report not receiving any advice from their ophthalmologist or optometrist regarding nutrition ${ }^{(10)}$. This may be partly due to the profession's differing opinions on nutrition research, but a patient's recall difficulties when given medical advice may also play a role.

\section{Other nutrients and energy}

With the exception of iron and protein, AMD participants did not meet the RDA of the nutrients analysed. AMD participants' energy intake was lower than recommended for their age-group and gender. This lowering of energy intake with increasing age has been found in other studies and some suggest that this is a physiological response that older adults exhibit in reaction to a decline in physical activity ${ }^{(21)}$. Some studies ${ }^{(22,23)}$, suggest that those living alone might consume fewer calories than those living with others, although we found no such relationship. A study into eating habits of older adults found that they eat more when they are presented with variety, and this variety is the key to increasing calorific consumption ${ }^{(24)}$. However, the non-AMD cohort managed to reach the RDA of many of the nutrients analysed, including calories. It may be argued that this was because there were fewer participants in the sample, and the average age of the sample was younger. However, when an age-matched cohort was analysed, the same differences in dietary intake was found. The non-AMD cohort did not eat a more varied diet either; they appeared to just eat more food than the AMD group. The cause of this is likely to also be multi-factoral; it has been shown in studies that poorer vision impacts on an individual's appetite ${ }^{(25,26)}$. More AMD participants were reliant on other people to shop and prepare and cook food, and this also may limit the amount they eat.

The Hertfordshire cohort study ${ }^{(27)}$ found that two patterns of diet are prevalent in the older adult group - a 'prudent' pattern that is characterised by fish, fruit, vegetables and wholemeal cereals, and a 'traditional' pattern characterised by vegetables, processed meat and puddings. The comparison between occupation and nutrients did not show any statistical trends in our results, but 
it was noted that $62 \%$ of AMD participants and $76 \%$ of non-AMD participants adhered to a 'traditional' pattern of eating with limited choices of vegetables and fruits, and traditional British recipes such as pies, stews and roast meats. This conservative variety of vegetables saw only a minority of AMD participants consuming the lutein-rich kale on a weekly basis, although more participants did eat spinach.

\section{Strengths and weaknesses}

This sample may not represent all AMD patients seeking services from organisations like the Macular Society. It is also important to find out the opinions of those with AMD who have not sought support from non-professional organisations. The sample sizes are different, and have been discussed. A more detailed food recall might have enhanced the study, especially to view eating patterns. This could be performed over a number of days rather than 24 hours. It would have been beneficial to gather other data such as participant's BMI and activity levels to compare to calorific intake. It is conceivable that a difference in BMI between the groups might have accounted for some of the dietary differences reported, but as this data was not collected, it is impossible to determine this. We feel that it is unlikely that differences between the groups are due to differences in BMI alone, as a previous study reported lower calorie intake in visually impaired participants ${ }^{(11)}$ and other studies show that food intake and dietary patterns do not appear to differ with various BMIs. (28-30)

Results show that participants were not in poor health generally, suggesting that results relate to issues with visual impairment only. Practitioners need to be consistent and unified in the advice that is given to patients, if confusion is to be avoided. The results also show that, in spite of advice being given to patients, they primarily eat food they enjoy and are used to. Changing eating habits therefore, requires novel intervention methods. It is essential to design effective measures for imparting and disseminating appropriate dietary and supplementation advice for patients with, or at risk of, AMD.

\section{Acknowledgments}

This study was conducted with support from the Macular Society, but not funded by the Macular Society.

\section{Statement of Authorship}


All three authors collaborated for the conception and design of the study. Rebekah Stevens analysed and interpreted much of the data, and drafted the article. Hannah Bartlett and Richard Cooke revised and approved the final version of the article to be published.

\section{Conflict of Interest and Funding Sources}

This research received no specific grant from any funding agency, commercial or not-for-profit sectors. We declare no conflicts of interest.

\section{$\underline{\text { References }}$}

1. RNIB. Age Related Macular Degeneration: RNIB and RCOphth; 2010. Available from: http://www.rnib.org.uk/eyehealth/eyeconditions/conditionsac/pages/amd.aspx.

2. Owen CG, Jarrar Z, Wormald R, Cook DG, Fletcher AE, Rudnicka AR. The estimated prevalence and incidence of late stage age related macular degeneration in the UK. BRITISH JOURNAL OF OPHTHALMOLOGY. 2012;96(5):752-6.

3. Shaw C. 2004-based national population projections for the UK and constituent countries. Population trends. 2006(123):9-20.

4. Kassoff A, Kassoff J, Buehler J, Eglow M, Kaufman F, Mehu M, et al. A randomized, placebocontrolled, clinical trial of high-dose supplementation with vitamins $C$ and $E$, beta carotene, and zinc for age-related macular degeneration and vision loss: AREDS report no. 8. Archives of Ophthalmology. 2001;119(10):1417-36.

5. Beatty S, Nolan J, Kavanagh H, O'Donovan O. Macular pigment optical density and its relationship with serum and dietary levels of lutein and zeaxanthin. Archives of Biochemistry and Biophysics.430:70-6.

6. Junghans A, Sies $\mathrm{H}$, Stahl W. Macular pigments lutein and zeaxanthin as blue light filters studied in liposomes. Archives of Biochemistry and Biophysics. 2001;391(2):160-4.

7. Chew EY, Clemons TE, SanGiovanni JP, Danis R, Ferris FL, Elman M, et al. Lutein plus Zeaxanthin and Omega-3 Fatty Acids for Age-Related Macular Degeneration The Age-Related Eye Disease Study 2 (AREDS2) Randomized Clinical Trial. Jama-Journal Of The American Medical Association. 2013;309(19):2005-15.

8. Kent C. AMD and Nutrition: The Missing Message. Review of Ophthalmology. 2007;14(8):31-7.

9. Perry A, Rasmussen H, Johnson EJ. Xanthophyll (lutein, zeaxanthin) content of fruits, vegetables and corn and egg products. J Food Com \& Anal. 2009; 22:9-15 .

10. Stevens R, Bartlett HE, Walsh R, Cooke R. Age-related macular degeneration patients' awareness of nutritional factors. British Journal of Visual Impairment. 2014;32 (2):77-92.

11. Bartlett $H$, Eperjesi $F$. Dietary analysis and patterns of nutritional supplement use in normal and age-related macular disease affected subjects: A prospective cross-sectional study. Nutrition Journal. 2004;3.

12. Kinshuck D. Diabetic Retinopathy: The Zimbabwe Hand Jive. 2012 [12/01/2014]. Available from: http://medweb.bham.ac.uk/easdec/prevention/portionsize.htm.

13. Bartlett H, Howells O, Eperjesi F. The Role of Macular Pigment Assessment in Clinical Practice: A Review Clinical and Experimental Optometry. 2010;93(5):300-8.

14. Kaipainen K, Payne CR, Wansink B. Mindless Eating Challenge: Retention, Weight Outcomes, and Barriers for Changes in a Public Web-Based Healthy Eating and Weight Loss Program. JOURNAL OF MEDICAL INTERNET RESEARCH. 2012;14(6):100-13.

15. Ley P. Communicating with patients: Improving communication, satisfaction and compliance. New York: Croom Helm; 1988. 210 p.

16. Kessels RPC. Patients' memory for medical information. Journal of the Royal Society of Medicine. 2003;96(5):219. 
17. McGuire LC. Remembering what the doctor said: Organization and adults' memory for medical information. Experimental Aging Research. 1996;22(4):403-28.

18. Bankoff SM, Sandberg EH. Older Adults' Memory for Verbally Presented Medical Information. Educational Gerontology. 2012;38(8):539-51.

19. Sandman L, Granger BB, Ekman I, Munthe C. Adherence, shared decision-making and patient autonomy. Medicine Health Care And Philosophy. 2012;15(2):115-27.

20. Ng WT, Goggin M. Awareness of and compliance with recommended dietary supplement among age-related macular degeneration patients. Clinical \& Experimental Ophthalmology. 2006;34(1):9-14.

21. Sarti S, Ruggiero E, Coin A, Toffanello ED, Perissinotto E, Miotto F, et al. Dietary intake and physical performance in healthy elderly women: A 3-year follow-up. Experimental Gerontology. 2013;48(2):250-4.

22. Gariballa SE, Forster SJ. Dietary intake of older patients in hospital and at home: the validity of patient kept food diaries. The Journal Of Nutrition, Health \& Aging. 2008;12(2):102-6.

23. Wright L, Hickson M, Frost G. Eating together is important: using a dining room in an acute elderly medical ward increases energy intake. Journal of Human Nutrition \& Dietetics. 2006;19(1):23-6.

24. Hollis JH, Henry CJK. Dietary variety and its effect on food intake of elderly adults. Journal of Human Nutrition \& Dietetics. 2007;20(4):345-51.

25. Lee JS, Kritchevsky SB, Tylavsky F, Harris TB, Ayonayon HN, Newman AB. Factors associated with impaired appetite in well-functioning community-dwelling older adults. Journal of Nutrition for the Elderly. 2007;26(1-2):27-43

26. Brennan M, Horowitz A, Su YP. Dual sensory loss and its impact on everyday competence. Gerontologist. 2005;45(3):337-46.

27. Robinson S, Syddall H, Jameson K, Batelaan S, Martin H, Dennison EM, et al. Current patterns of diet in community-dwelling older men and women: results from the Hertfordshire Cohort Study. Age And Ageing. 2009;38(5):594-9.

28. Kimokoti RW, Judd SE, Shikany JM, Newby PK. Food Intake Does Not Differ between Obese Women Who Are Metabolically Healthy or Abnormal. Journal of Nutrition. 2014;144(12):2018.

29. Murakami K, Sasaki S, Okubo H, Takahashi Y. Neighborhood socioeconomic status in relation to dietary intake and body mass index in female Japanese dietetic students. Nutrition. 2009;25(7/8):745-52.

30. Togo P, Osler M, Sørensen TIA, Heitmann BL. Food intake patterns and body mass index in observational studies. International Journal of Obesity \& Related Metabolic Disorders. 2001;25(12):1741. 


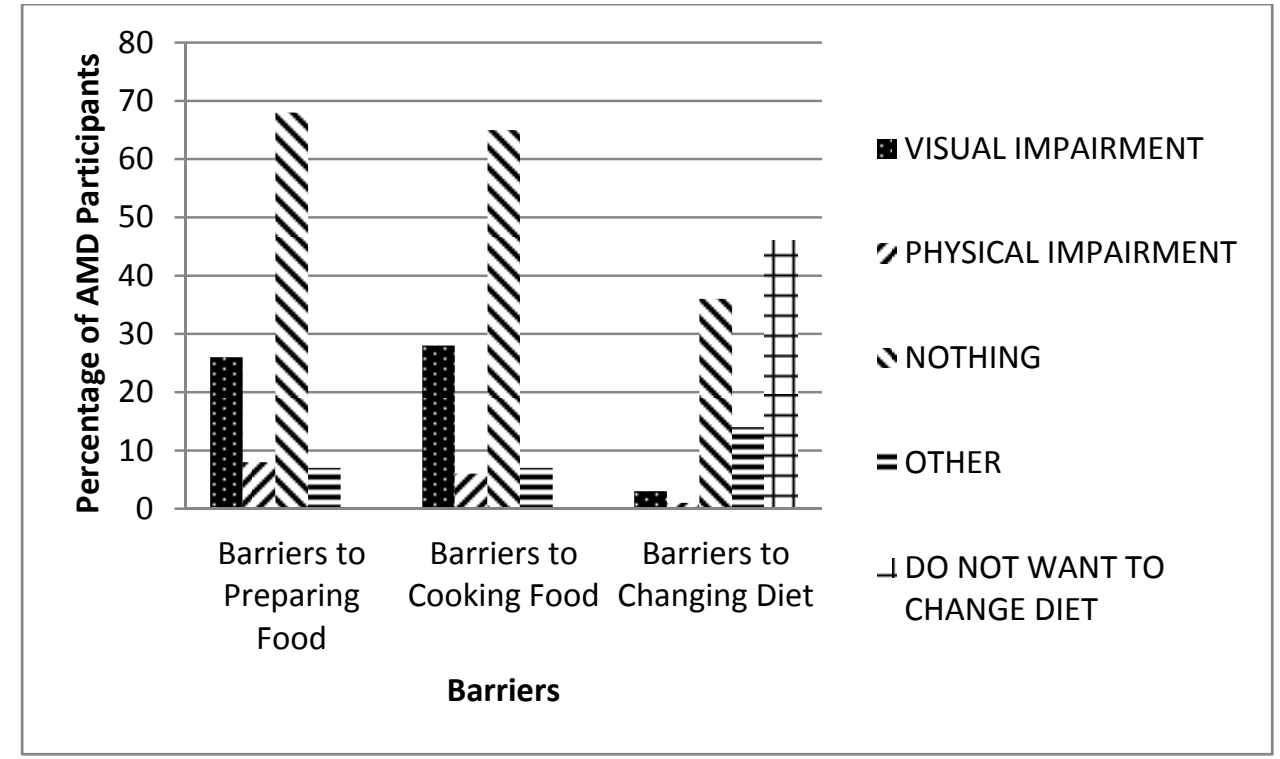

Figure 1. AMD participants perceived barriers to cooking food, preparing food and changing diet. 


\begin{tabular}{|c|c|c|c|}
\hline Characteristic & Characteristic & $\begin{array}{l}\text { Percentage of AMD } \\
\text { Participants }\end{array}$ & $\begin{array}{l}\text { Percentage of Non-AMD } \\
\text { Participants }\end{array}$ \\
\hline \multirow[t]{4}{*}{$\begin{array}{l}\text { Living } \\
\text { Arrangements }\end{array}$} & Own home & $88 \%$ & $96 \%$ \\
\hline & With family/friends & $3 \%$ & 0 \\
\hline & Sheltered accommodation & $7 \%$ & $4 \%$ \\
\hline & Other & $2 \%$ & 0 \\
\hline \multirow[t]{3}{*}{ With } & Partner & $50 \%$ & $66 \%$ \\
\hline & Alone & $46 \%$ & $30 \%$ \\
\hline & Other family members & $4 \%$ & $4 \%$ \\
\hline \multirow[t]{3}{*}{ Registration } & Blind & $16 \%$ & Not Applicable \\
\hline & Partially sighted & $21 \%$ & Not Applicable \\
\hline & None & $63 \%$ & Not Applicable \\
\hline \multirow[t]{5}{*}{$\begin{array}{l}\text { General } \\
\text { Health }\end{array}$} & Extremely good & $7 \%$ & $22 \%$ \\
\hline & Good & $41 \%$ & $48 \%$ \\
\hline & Satisfactory & $19 \%$ & $20 \%$ \\
\hline & Poor & $21 \%$ & $8 \%$ \\
\hline & Extremely poor & $3 \%$ & $2 \%$ \\
\hline \multirow[t]{4}{*}{ Vision } & Extremely good & $1 \%$ & $14 \%$ \\
\hline & Good & $7 \%$ & $46 \%$ \\
\hline & Satisfactory & $35 \%$ & $32 \%$ \\
\hline & Poor & $42 \%$ & $8 \%$ \\
\hline
\end{tabular}




\begin{tabular}{|l|l|l|l|}
\hline & Extremely poor & $15 \%$ & 0 \\
\hline
\end{tabular}

3 Table 1. Selected demographic characteristics of both AMD and non-AMD participants.

\begin{tabular}{|c|c|c|c|c|c|c|c|}
\hline & & $\begin{array}{l}\text { AMD } \\
\text { Female } \\
(\text { Mean) } \\
(\mathrm{n}=96)\end{array}$ & $\begin{array}{l}\text { AMD } \\
\text { Male } \\
\text { (Mean) } \\
(n=62)\end{array}$ & $\begin{array}{l}\text { Non- } \\
\text { AMD } \\
\text { Female } \\
\text { (Mean) } \\
(n=35)\end{array}$ & $\begin{array}{l}\text { Non- } \\
\text { AMD } \\
\text { Male } \\
(\text { Mean) } \\
(n=15)\end{array}$ & $\begin{array}{l}\text { Females } \\
\text { RDA } \\
>50 \mathrm{yrs}\end{array}$ & $\begin{array}{l}\text { Males } \\
\text { RDA } \\
>50 \mathrm{yrs}\end{array}$ \\
\hline Energy & Kcal & 1524 & 1507 & 2251 & 2074 & 1800 & 2200 \\
\hline Energy & $\mathrm{kJ}$ & 6375 & 6076 & 9417 & 8674 & 7200 & 8700 \\
\hline Lutein and Zeaxanthin & $\mathrm{Mg}$ & 1.7 & 1.2 & 1.6 & 1.3 & 10 & 10 \\
\hline Protein & G & 61.8 & 64.1 & 94.5 & 82.0 & 45 & 55 \\
\hline Fat & G & 65.4 & 60.5 & 86.8 & 82.3 & 70 & 95 \\
\hline of which saturates & G & 27.7 & 25.4 & 30.8 & 30.6 & 20 & 30 \\
\hline Carbohydrates & G & 177.2 & 169.0 & 278.6 & 257.5 & 230 & 300 \\
\hline of which sugars & G & 50.5 & 44.7 & 90.9 & 61.8 & 90 & 120 \\
\hline Fibre & G & 16.7 & 16.1 & 24.6 & 22.4 & 24 & 24 \\
\hline Cholesterol & $\mathrm{Mg}$ & 252.9 & 300.8 & 354.6 & 408.0 & 300 & 300 \\
\hline Calcium & $\mathrm{Mg}$ & 682.9 & 644.0 & 948.0 & 980.5 & 800 & 800 \\
\hline Iron & $\mathrm{Mg}$ & 14.5 & 16.1 & 22.4 & 20.4 & 10 & 10 \\
\hline Retinol & $\mathrm{mg}$ & 0.4 & 0.4 & 0.4 & 0.6 & & \\
\hline Carotene & $\mathrm{mg}$ & 0.4 & 0.7 & 0 & 0 & & \\
\hline Alpha Carotene & $\mathrm{mg}$ & 1.1 & 1.4 & 1.1 & 1.3 & & \\
\hline Beta Carotene & $\mathrm{mg}$ & 3.5 & 4.1 & 3.8 & 4.0 & & \\
\hline Vitamin D & $\mu g$ & 2.6 & 13.5 & 2.4 & 3.5 & 5 & 5 \\
\hline Vitamin E & $\mathrm{Mg}$ & 5.0 & 5.1 & 7.6 & 6.0 & 10 & 10 \\
\hline Vitamin C & $\mathrm{Mg}$ & 53.1 & 62.1 & 85.6 & 82.3 & 40 & 40 \\
\hline Folic Acid & $\mu \mathrm{g}$ & 167.8 & 252.2 & 214.4 & 185.7 & 400 & 400 \\
\hline
\end{tabular}

5 
7

\begin{tabular}{lrr}
\hline & AMD Sub-Group & \multicolumn{2}{c}{ Non-AMD } \\
& Mean (n=49) & \multicolumn{2}{c}{ Mean ( $=50)$} \\
\hline Age (years) & 68.8 & 67.5 \\
Energy (Kcal) & 1503 & 2198 \\
L\&Z (mg) & 1.8 & 1.5 \\
Protein (G) & 60.9 & 90.8 \\
Fat (G) & 61.9 & 85.5 \\
Carbohydrates (G) & 166.9 & 272.3 \\
Fibre (G) & 15.6 & 24 \\
Vitamin E (mg) & 5.3 & 7.1 \\
Beta Carotene ( $\mu$ g) & 3.2 & 3.9 \\
Vitamin C (mg) & 56.9 & 84.6 \\
Folic Acid (mg) & 0.2 & 0.21 \\
Calcium (mg) & 596.1 & 887.8 \\
\hline
\end{tabular}
$\mathrm{G}$ refers to grams. Spaces indicate there are no RDAs for that nutrient 3 7 micrograms, Mg refers to miligrams, and G refers to grams.

Table 2. Mean consumption of various nutrients for males and females in both the AMD cohort and the non-AMD cohort, with the corresponding RDA for people aged over 50 years. Please note: Kcal refers to Kilocalories, kJ Refers to Kilojoules, Mg refers to miligrams, $\mu \mathrm{g}$ refers to micrograms and

Table 3. Mean age and energy/ nutrient consumption for the selected AMD subgroup and the nonAMD group. Please note: Kcal refers to Kilocalories, kJ Refers to Kilojoules, $\mu$ g refers to 